\title{
Family Meetings Move Online
}

\author{
Katelyn Husereau (CFAR)
}

KEYWORDS: Information Technology, Family

Business, crisis management.

Family enterprises have been impacted in many ways by COVID-19, with many families placing their focus on the core business both in order to survive and to take advantage of this time to put forth new initiatives that had been put on the back burner.

In the face of these unprecedented circumstances, businesses have had to make sharp, critical pivots to account for threats to their core business, while having to increase safety protocols, establish new operating procedures, and implement and rely on new technology-all under incredible circumstances and financial pressures. While this focus on keeping the business going in the short term is critical, the family enterprises that will make it through this moment and beyond also have their eye on the long term, focusing on business and on the family.

A key facet of both business and family engagement, naturally, is meeting face to face; however, the reality of COVID-19 dictates that doing so in-person is largely not worth the risk. As a result, families have had to get creative in order to maintain connection with one another. Virtual meetings have become ubiquitous-hosted on Zoom, WebEx, etc.-but do these technologies support the same kind of connection as an in-person gathering? Do virtual meetings provide a safe and viable alternative to in-person meetings? Of course, our short answer is yes! While it can be done, don't trick yourself into thinking it is simple to move from in-person to online-it requires a whole different approach along with deliberate effort and planning. Beginning in March of last year, we started to see families embrace this transition and, since that time, we have learned lessons about what works and what doesn't when it comes to these virtual family meetings. In this article, we're sharing some of the lessons learned and factors to consider when thinking about hosting a virtual family meeting.

\section{The Silver Lining of Virtual}

\section{Meetings}

The primary benefit of virtual meetings is the ease of convening (especially for large families). We have all had to coordinate dates and puzzle-piece through distance, travel, and logistics to convene a traditional, inperson family gathering. The frenzy of the planning itself can distract from the very reason a family is getting together in the first place-to connect, to inform, and to learn together. Attention to logistics is always crucial, but the relative ease of convening virtual meetings allows you to re-channel your energy towards meeting design (agendas, goals, and results), material creation, and technological considerations rather than worrying about things like how the Smiths will be getting to Florida, when Maggie will be on school break, and what food allergies and preferences you have to account for.

We've also found that families who traditionally manage to only have $75 \%$ of family members present for inperson gatherings are now experiencing nearly $100 \%$ participation-a result of the increased ease of attendance. To focus deeper on who is showing up and how they're showing up, we've found that it's possible to increase engagement from next-generation members given their comfort and familiarity with the video platforms used for most meetings. This yields greater participation and contributions from an age group that may otherwise feel out of its element amid large, in-person meetings. Zoom also helps level the playing field-with everyone having an equal share of the screen and position in the room within the virtual "boxes" they occupy.

Virtual formats also make it easier to break meetings up into bite-sized, digestible chunks, which reduces meeting fatigue and can help sustain the attention and focus of family members. Instead of a full-day gathering (involving activities and content for eight hours straight, with dinners and activities in between), many families have opted out of adapting to full-day eight-hour video meetings (understandably so), instead choosing to hold
Copyright @ 2021 The Authors. Entrepreneur \& Innovation Exchange is published at EIX.org. This is an open access article under the terms of the Creative Commons Attribution-NoDerivs License, which permits use and distribution in any medium, provided the original work is properly cited and no modifications or adaptations are made. View EIX.org Authorship Terms at https://eix.org/terms
FamilyBusiness 
multiple 90-minute meetings over the course of a couple of months. With the right amount of follow-up and confirmed next steps in place, this meeting cadence can also work to support the goal of creating continuity and connection between sessions.

Lastly, families are taking advantage of this turbulent time to advance important work that they may have been thinking about for years but haven't had the push to do. Successful families are calling on the multiple talents in the family in a time of crisis to tackle issues in three months that before took three years. Many families are coming together in this time of urgency and opportunity to advance long-term agendas in short periods of time.

\section{Tips, Tricks, and Best Practices}

Below are a few of our key best practices to keep in mind when executing virtual family meetings, including how to structure and sequence conversations and some technical tips for making them happen.

1. Plan virtual logistics like you would plan for a physical meeting (but without those extra distractions - food, travel, etc.). Cross your Ts and dot your Is. For example, make sure everyone can access the meeting (this may mean doing a trial run with those less familiar with the platform); make sure meeting materials are ready and accessible and that any videos or documents you might want to share can be accessed and viewed. You can even use virtual meetings as an opportunity to engage family members who might not have seen a role for themselves before. For example, maybe a younger generation member has been taking classes virtually through Zoom and can help with the technical components to make sure things run smoothly. You will also want to make sure that meeting roles are clarified. For example-who can family members call if they have any technological challenges, who is sharing the screen, who is taking notes and capturing follow-ups, etc.?

2. Break it up! The beauty of virtual meetings is that you don't have to rely on people being all together (like past meetings), which means you can spread sessions across a longer amount of time. As noted above, we recommend sessions be no longer than 90 minutes with at least one short break. Many families choose to develop a series of sessions with cumulative content while others prefer stand-alone sessions.

3. Steal your best practices from in-person meetings and be explicit about your expectations and meeting ground rules. You may consider addressing expectations on video use, muting, how to use the chat, how people interact (hand raising or jump in), etc. Think about content, breaks, as well as bringing in different voices, discussion leads, or presenters-even special guests or outside speakers, given the ease of having people join in and hop off.

4. Make content interactive with opportunities for engagement. It's easy in virtual meetings to focus solely on content and forget to leave room for discussion and engagement. There are many ways to make virtual meetings interactive. We recommend creating structures for conversation and engagement. For example, query the full group in the chat window, send out a poll to get real-time data, or craft a virtual group game or activity to lighten the mood. You can also use breakout rooms to give family members an opportunity to hold more intimate conversations in smaller groups with less voice competition. The hope is to give family members the opportunity to laugh, share, and have fun together while staying informed and keeping connected.

5. Consider using facilitators. Now is a time to enlist expert help to support engagement of the family, and develop the capabilities, processes, and supports you need to sustain family connection and development into the future.

\section{In Sum}

At the end of the day, Zoom and other virtual technologies are no replacement for seeing your relatives in person and sharing an embrace. On the other hand, the circumstances of COVID-19 force us to postpone those intimate moments until we can feel safe doing so once more. As such, the tools at our disposal become indispensable not only for the purposes of business, but also for continuing to cultivate the relationships most important to us. 